\title{
Predictors for early mortality and arrhythmic events in patients with cardiac resynchronization therapy with defibrillator: A two center cohort study
}

\author{
Simon von Gunten ${ }^{1}$, Dominic A. Theuns ${ }^{2}$, Michael Kühne ${ }^{1}$, Tobias Reichlin ${ }^{1}$, \\ Christian Sticherling ${ }^{1}$, Beat Schaer ${ }^{1}$ \\ ${ }^{1}$ Department of Cardiology, University Hospital, Basel, Switzerland \\ ${ }^{2}$ Department of Cardiology, Erasmus Medical Center, Rotterdam, The Netherlands
}

\begin{abstract}
Background: Guidelines of heart failure therapy include cardiac resynchronization as standard of care in patients with severely depressed left ventricular function and wide QRS complex. It has been shown that patients benefit regarding mortality and morbidity. However, early mortality precludes longterm benefits from the device. The aim of the study was to identify predictors for early occurrence of both death and first-ever implantable cardioverter-defibrillator (ICD) therapy using a large combined database of patients with cardiac resynchronization therapy with defibrillator (CRT-D).

Methods: From two registries (tertiary care centers) 904 patients were identified, no single patient was excluded. Early death was defined as death occurring within the 3 years after implantation whereas early ICD therapy as such occurring within the first year. 33 baseline parameters were compared using uni-and multivariate analysis with the Cox model and binary logistic regression.

Results: The population was predominantly male (77\%), with mean age of $63 \pm 11$ years and primary prevention indication in 80\%. Mean follow-up was $55 \pm 38$ months. 256 (28\%) patients had ICD therapies whereof the first-ever event occurred early in 52\%. 270 (30\%) patients died after $41 \pm$ \pm 31 months, mostly from advancing heart failure (41\%), 141 (52\%) patients of them early. Independent predictors for early ICD therapy were secondary prevention and renal failure. Independent predictors for early mortality were a history of percutaneous coronary intervention and of peripheral vascular disease.

Conclusions: Predictors for early mortality after CRT-D implantation were a history of percutaneous coronary intervention and peripheral vascular disease, present in only a minority of patients, thus limiting their use in clinical practice. (Cardiol J 2019; 26, 6: 711-716)

Key words: cardiac resynchronization therapy, implantable cardioverter-defibrillator, mortality, predictive model, decision making
\end{abstract}

\section{Introduction}

Implantation of a cardiac resynchronization therapy (CRT) device is standard of care in the therapy of heart failure patients with severely depressed left ventricular function and a wide QRS complex. Several randomized controlled trials have shown remarkable benefits of CRT regarding mortality and morbidity in combination with an implantable cardioverter-defibrillator (ICD) but also with a stand-alone pacemaker [1,2]. Many patients present with left ventricular ejection fraction (LVEF) below 35\%, and thus are implanted with a CRT defibrillator (CRT-D). However, no strong evidence suggests that CRT-D must be used in all patients that are CRT candidates [3]. A relevant number of patients have severe comorbidities and die early after implant. Therefore, they might not

Address for correspondence: Prof. Beat Schaer, MD, Department of Cardiology, Petersgraben 4, CH 4031 Basel, Switzerland, tel: +416132862 22, fax: +416126545 80, e-mail: beat.schaer@usb.ch 
be in need of the ICD component of a CRT-D $[4,5]$. Identification of such patients is considered important, as death as the competing event obviously precludes potential long-term benefit from the ICD component of CRT. Data regarding first-ever ICD therapy are conflicting; studies have shown both linear and asymptotic event curves [6-8]. The aim of this study was to determine independent predictors for early occurrence of both death and first-ever ICD therapy. If meaningful and/or highly prevalent predictors were identified, they could help in decision making for CRT-D or for CRT-pacemaker (CRT-P).

\section{Methods}

The study population consisted of all consecutive patients in whom a CRT-D was implanted at the Erasmus Medical Center, Rotterdam, the Netherlands or at the University of Basel Hospital, Switzerland. At the sites, patients are entered into separate registries that were started in Rotterdam in November $1999(\mathrm{n}=608$ patients $)$ and in Basel in February 2000 ( $n=296$ patients). Last access to the database was July 2015 for Rotterdam patients and May 2015 for Basel patients. Data merging was performed in August 2015. No patients were excluded, leading to a total amount of 904 patients.

Deaths were classified as being due to progressive heart failure, clearly non-cardiac causes, sudden (i.e. a sudden death without post mortem analysis of the CRT-D and/or necropsy) or arrhythmic (i.e. the device could be interrogated showing either ventricular fibrillation that could not have been terminated by all shocks or sinus rhythm after successful shocks with subsequent electromechanical dissociation).

Patient and device characteristics are recorded prospectively at baseline, including 33 parameters such as demographic and cardiovascular items, comorbidities, drugs and laboratory values. Missing values of LVEF of 66 patients from Rotterdam were imputed as well as 5 missing values of blood urea nitrogen levels, and 2 values of $\mathrm{QRS}$ width and sodium, respectively, using the median of each parameter [9]. Renal failure was defined as glomerular filtration rate $(\mathrm{GFR})<60 \mathrm{~mL} / \mathrm{min} / 1.73 \mathrm{~m}^{2}$. Appropriate ICD therapy are considered in the ventricular tachycardia (VT) zone of the ICD (tachycardia of $180-220 / \mathrm{min}$, primarily terminated by antitachycardia pacing [ATP] or cardioversion shock if ATP failed) and in the ventricular fibrillation (VF) zone (tachycardias $>220 /$ min terminated by ATP during charging or by cardioversion shock). True ventricular fibrillation terminated by defibrillation was studied separately. In cases where ATP accelerated the VT into the VF zone, the initial VT was considered as the event of interest. The first-ever ICD therapy was defined as early when occurring within 12 months after implantation (median time of first-ever ICD therapy). Death was defined as early when occurring within 3 years after implantation (median time of death). All 33 baseline parameters were compared in univariate analysis for both events.

\section{Statistical analysis}

Continuous variables are presented as mean \pm standard deviation or median with interquartile range and categorical variables as numbers and percentages. To determine the prognostic impact of significant variables, univariate Cox regression and binary logistic regression were used to compute hazard ratios (respectively odds ratios [ORs]) and $95 \%$ confidence intervals (CIs). All variables predicting death or ICD therapies significantly with a $\mathrm{p}$ value of $\leq 0.1$ in the univariate model were entered in a multivariate model using the forward stepwise method. Statistical analyses were performed with the use of IBM SPSS Statistics for Windows, version 23.0 (SPSS Inc. Chicago, IL).

The study is a retrospective merged analysis of data collected prospectively in both centers.

\section{Results}

\section{Baseline characteristics and follow-up}

A merged population of 904 patients was predominantly male $(77 \%)$, had a mean age of $63 \pm 11$ years at implant and a primary prevention indication in $80 \%$. Mean follow-up was $55 \pm 38$ months. Table 1 depicts all relevant baseline characteristics in detail. Figure 1 shows a flow chart of patients included in the study, mortality rate and mode of death, the arrhythmias treated by the ICD, and their separation into early or non-early events.

\section{ICD therapies}

During follow-up, 256 (28\%) patients had ICD therapies. The first-ever event occurred early in 134 (52\%) patients. In 2/3 ICD, therapy was delivered in the VT zone (82\% of them occurring early), in $1 / 3$, in the VF zone of the ICD (77\% of them occurring early). Independent predictors for early ICD therapy were secondary prevention (OR 3.21, 1.84-5.56, 0.01 ) and renal failure (OR 2.08, 1.24-3.50, 0.01). More detailed data is presented in Tables 2 and 3. 
Table 1. Baseline characteristics of the 904 patients.

\begin{tabular}{|c|c|}
\hline Male gender & $700(77 \%)$ \\
\hline Age at implant [years] & $63 \pm 11$ \\
\hline Weight [kg] & $82 \pm 16$ \\
\hline Body mass index & $27 \pm 5$ \\
\hline Systolic BP [mmHg] & $112 \pm 19$ \\
\hline Ejection fraction [\%] & $25 \pm 7$ \\
\hline QRS width [ms] & $163 \pm 29$ \\
\hline Primary prevention & $720(80 \%)$ \\
\hline Sinus rhythm & $793(88 \%)$ \\
\hline NYHA I class & $8(1 \%)$ \\
\hline NYHA II class & $297(33 \%)$ \\
\hline NYHA III class & $571(63 \%)$ \\
\hline Ambulatory NYHA IV class & $28(3 \%)$ \\
\hline Clearance $\left[\mathrm{mL} / \mathrm{min} / 1.73 \mathrm{~m}^{2}\right]$ & $64 \pm 25$ \\
\hline $\begin{array}{l}\text { Renal failure } \\
\text { (GFR }<60 \mathrm{~mL} / \mathrm{min} / 1.73 \mathrm{~m}^{2} \text { ) }\end{array}$ & $434(48 \%)$ \\
\hline Ischemic cardiomyopathy & $451(50 \%)$ \\
\hline Myocardial infarction & $371(41 \%)$ \\
\hline $\mathrm{PCl}$ & $218(24 \%)$ \\
\hline CABG & $191(21 \%)$ \\
\hline Diabetes mellitus & $224(25 \%)$ \\
\hline Stroke & $112(12 \%)$ \\
\hline COPD & $107(12 \%)$ \\
\hline Cancer & $70(8 \%)$ \\
\hline PVD & $62(7 \%)$ \\
\hline \multicolumn{2}{|l|}{ Drug therapy: } \\
\hline Diuretics & $758(84 \%)$ \\
\hline ACE-inhibitors & $751(83 \%)$ \\
\hline Beta-blockers & $736(81 \%)$ \\
\hline Statins & $508(56 \%)$ \\
\hline Aldosterone antagonists & $416(46 \%)$ \\
\hline Angiotensin receptor blockers & $216(24 \%)$ \\
\hline Amiodarone & $202(22 \%)$ \\
\hline Digoxin & $189(21 \%)$ \\
\hline Allopurinol & $71(8 \%)$ \\
\hline Sodium $[\mathrm{mmol} / \mathrm{L}]$ & $139 \pm 4$ \\
\hline Hemoglobin $[g / L]$ & $134 \pm 18$ \\
\hline Blood urea nitrogen [mmol/L] & $10 \pm 6$ \\
\hline
\end{tabular}

$\mathrm{ACE}$ - angiotensin converting enzyme; $\mathrm{BP}$ — blood pressure; CABG - coronary artery bypass grafting; COPD — chronic obstructive pulmonary disease; GFR - glomerular filtration rate; NYHA - New York Heart Association; $\mathrm{PCl}$ - percutaneous coronary intervention; PVD - peripheral vascular disease

Independent predictors for VT as compared to fast VT/VF were beta-blocker therapy (OR 1.77, 1.09$-2.86,0.02$ ) and increasing hemoglobin level (hazard ratio per $1 \mathrm{~g} / \mathrm{L} 1.02,1.01-1.04,0.01)$.

\section{Mortality}

Overall, $270(30 \%)$ patients died after a mean $41 \pm 31$ months, mostly from advancing heart failure $(41 \%)$ or non-cardiac causes (18\%). Of note, the cause of death (not the time of death) was not recorded in the dataset in 33\%. $141(52 \%)$ patients had died within the first 3 years and thus early. Independent predictors for early mortality were a history of percutaneous coronary intervention (PCI) (OR 2.01, 1.10-3.66, 0.02) and peripheral vascular disease (PVD) (OR 2.91, 1.08-7.87, 0.04). However, only $24 \%$ of patients had a history of PCI and $7 \%$ of PVD. A more detailed overview is shown in Table 4.

\section{Discussion}

During a mean follow-up of more than 5.5 years, only $28 \%$ of these CRT-D patients had appropriate ICD therapies. The first-ever event occurred early in $52 \%$. A third of them were delivered for potentially life-threatening arrhythmias. This rate is slightly lower than the mortality of these severely sick patients (50\% renal failure, $66 \%$ in the New York Heart Association [NYHA] class III or ambulatory class IV). With the inclusion of 33 parameters to study early ICD therapy and early mortality, it was determined that secondary prevention and renal failure were predictors for early ICD therapy and history of PCI and PVD for early mortality.

It would be both scientifically intriguing and clinically helpful to identify those patients who either die early after ICD implantation (and in whom CRT-D implantation can be questioned and CRT-P offered) or those who never experience ICD therapies. It is thus not surprising that several studies have been undertaken with the focus of early mortality $[4,6,10,11]$. In the present study only history of PCI and PVD were identified as predictors for early mortality, i.e. at 3 years. However, the clinical application of them has to be questioned, as they were present in only $24 \%$ and $7 \%$ of patients, respectively. In addition, a sub-analysis from MADIT-CRT showed that a history of PCI, independent of frequency or timing before enrolment, did not influence mortality [12]. Finally, there is no intuitive explanation for these two identified predictors and, due to the low number of patients with these comorbidities, a type I error especially for PVD cannot be excluded. In summary, this study failed to fulfil the particular aim of predicting early mortality in a clinically applicable way. 


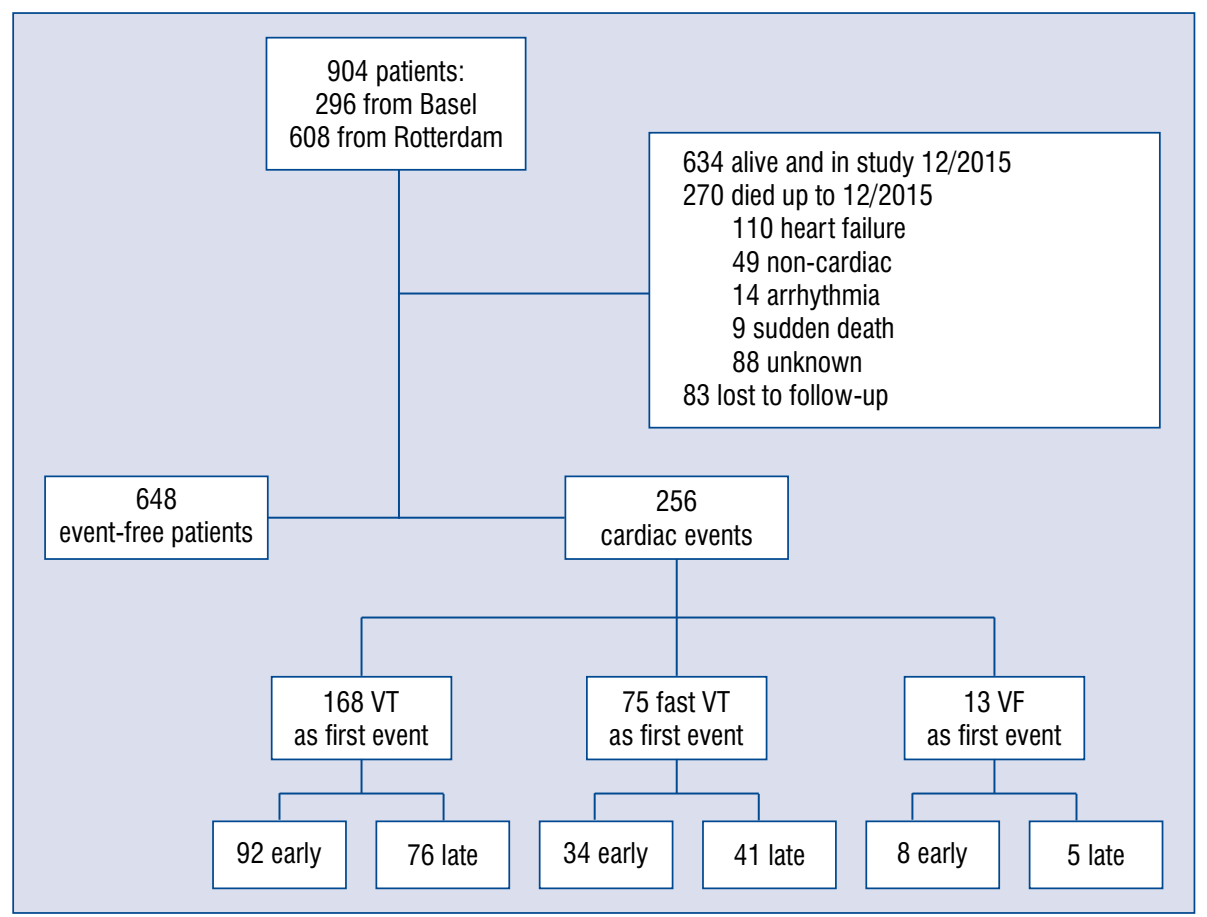

Figure 1. Flow chart of patients and cardiac events; VT — ventricular tachycardia (> 180/min); fast VT — fast ventricular tachycardia (> 220/min); VF — true ventricular fibrillation.

Table 2. Univariable and multivariable analysis for occurrence of first-ever implantable cardioverterdefibrillator therapy without temporal discrimination (only significant parameters are shown, hazard ratio $<1=$ no cardiac event, hazard ratio $>1=$ cardiac event).

\begin{tabular}{|c|c|c|c|c|c|c|}
\hline & \multicolumn{3}{|c|}{ Univariable analysis } & \multicolumn{3}{|c|}{ Multivariable analysis } \\
\hline & HR & $95 \% \mathrm{Cl}$ & $\mathbf{P}$ & HR & $95 \% \mathrm{Cl}$ & $\mathbf{P}$ \\
\hline Secondary prevention & 1.596 & $1.232-2.068$ & 0.000 & 1.534 & $1.183-1.990$ & 0.001 \\
\hline Renal failure & 1.468 & $1.142-1.888$ & 0.003 & 1.408 & $1.093-1.814$ & 0.008 \\
\hline Amiodarone therapy & 1.423 & $1.075-1.883$ & 0.014 & - & & \\
\hline Clearance $\left[\mathrm{mL} / \mathrm{min} / 1.73 \mathrm{~m}^{2}\right]$ & 0.993 & $0.987-0.999$ & 0.014 & - & & \\
\hline Age at implant [year] & 1.015 & $1.005-1.026$ & 0.004 & - & & \\
\hline Blood urea nitrogen [mmol/L] & 1.022 & $0.998-1.046$ & 0.074 & - & & \\
\hline
\end{tabular}

$\mathrm{Cl}$ - confidence interval; $\mathrm{HR}$ - hazard ratio

In CRT-D patients, no similar data are available to date. In a large ICD only cohort study with a validation cohort (total patient number $2700,75 \%$ primary prevention) [10], four factors predicted mortality at 1 year. They were PVD, age $>70$ years, LVEF $<20 \%$ and creatinine $>176 \mathrm{mmol} / \mathrm{L}$. Patients with only one factor had a mortality of $4 \%$ as compared to $18 \%$ in those with four factors. Results were confirmed in a population of 800 patients (100\% primary prevention, 28\% CRT-D) [4]. Age (here: $>75$ years), impaired renal function (here:
GFR $\left.<30 \mathrm{~mL} / \mathrm{min} / 1.73 \mathrm{~m}^{2}\right)$, QRS width $>120 \mathrm{~ms}$, and atrial fibrillation were the four predictors. Mortality at 1 year was $2.5 \%$ in patients with 0 or 1 risk factors, but $46 \%$ in those with all four factors present. However, both papers do not discuss the fact that only $2 \%$ of patients were labelled as very high-risk patients, which renders the usefulness of these impressive results less applicable in daily life.

In a study of 225 octogenarians, LVEF $<20 \%$ and lack of beta-blocker therapy were the only two 
Table 3. Uni- and multivariable analyses for occurrence of first-ever implantable cardioverter-defibrillator therapy with discrimination between early and late events (only significant parameters are shown, odds ratio $<1=$ early [ $\leq 12$ months], odds ratio $>1=$ late [ $>12$ months]).

\begin{tabular}{|c|c|c|c|c|c|c|}
\hline & \multicolumn{3}{|c|}{ Univariable analysis } & \multicolumn{3}{|c|}{ Multivariable analysis } \\
\hline & OR & $95 \% \mathrm{Cl}$ & $\mathbf{P}$ & OR & $95 \% \mathrm{Cl}$ & $\mathbf{P}$ \\
\hline Secondary prevention & 0.302 & $0.175-0.521$ & 0.000 & 0.312 & $0.180-0.543$ & 0.000 \\
\hline Renal failure & 0.457 & $0.277-0.754$ & 0.002 & 0.480 & $0.286-0.807$ & 0.006 \\
\hline Amiodarone therapy & 0.623 & $0.355-1.092$ & 0.098 & & & - \\
\hline ARB therapy & 1.671 & $0.935-2.987$ & 0.083 & & & - \\
\hline Age at implant [year] & 0.969 & $0.948-0.990$ & 0.005 & & & - \\
\hline Weight [kg] & 1.018 & $1.001-1.034$ & 0.036 & & & - \\
\hline Blood urea nitrogen [mmol/L] & 0.951 & $0.906-0.998$ & 0.040 & & - & \\
\hline Clearance $\left[\mathrm{mL} / \mathrm{min} / 1.73 \mathrm{~m}^{2}\right]$ & 1.016 & $1.005-1.027$ & 0.006 & & - & \\
\hline Hemoglobin $[\mathrm{g} / \mathrm{L}]$ & 1.015 & $1.002-1.029$ & 0.029 & & & - \\
\hline
\end{tabular}

$\mathrm{ARB}$ - angiotensin receptor blocker; $\mathrm{Cl}$ - confidence interval; OR — odds ratio

Table 4. Uni- and multivariable analyses for occurrence of early death (only significant parameters are shown, odds ratio $<1=$ early $[\leq 36$ months], odds ratio $>1=$ late [ $>36$ months $]$ ).

\begin{tabular}{|c|c|c|c|c|c|c|}
\hline & \multicolumn{3}{|c|}{ Univariable analysis } & \multicolumn{3}{|c|}{ Multivariable analysis } \\
\hline & OR & $95 \% \mathrm{Cl}$ & $\mathbf{P}$ & OR & $95 \% \mathrm{Cl}$ & $\mathbf{P}$ \\
\hline Ischemic cardiomyopathy & 0.651 & $0.396-1.071$ & 0.091 & - & & \\
\hline $\mathrm{PCl}$ & 0.556 & $0.318-0.973$ & 0.040 & 0.498 & $0.273-0.908$ & 0.023 \\
\hline Diabetes mellitus & 0.639 & $0.376-1.086$ & 0.098 & - & & \\
\hline PVD & 0.313 & $0.121-0.811$ & 0.017 & 0.344 & $0.127-0.930$ & 0.035 \\
\hline Renal failure & 0.440 & $0.265-0.729$ & 0.001 & - & & \\
\hline Digoxin therapy & 0.600 & $0.354-1.018$ & 0.058 & - & & \\
\hline Weight [kg] & 1.019 & $1.003-1.035$ & 0.019 & - & & \\
\hline Body mass index [Unit] & 1.051 & $1.000-1.104$ & 0.050 & - & & \\
\hline Systolic BP [mmHg] & 1.012 & $0.999-1.024$ & 0.073 & 1.015 & $1.002-1.029$ & 0.025 \\
\hline Blood urea nitrogen [mmol/L] & 0.963 & $0.926-1.000$ & 0.050 & - & & \\
\hline Clearance $\left[\mathrm{mL} / \mathrm{min} / 1.73 \mathrm{~m}^{2}\right]$ & 1.018 & $1.007-1.029$ & 0.001 & 1.020 & $1.009-1.031$ & 0.001 \\
\hline Hemoglobin $[\mathrm{g} / \mathrm{L}]$ & 1.013 & $0.999-1.026$ & 0.060 & - & & \\
\hline
\end{tabular}

$\mathrm{BP}$ — blood pressure; $\mathrm{Cl}$ — confidence interval; OR — odds ratio; $\mathrm{PCl}$ - percutaneous coronary intervention; PVD — peripheral vascular disease

predictors of mortality at 1 year. Mortality of patients with LVEF $<20 \%$ was threefold compared to those with LVEF $>20 \%$, but the patient number at risk are not mentioned, thus severely limiting this statement. Finally, the Italian IRIDE registry [6] reported their results on 600 patients with primary prevention (43\% CRT-D). They showed a linear increase of mortality to $24 \%$ at 4 years, which is similar to the present results. This is surprising, given the much higher disease burden in the current study (48\% renal failure and $71 \%$ NYHA class
III/IV vs. $11 \%$ and $38 \%$, respectively). In addition, even though ICD programing is comparable, the rate of ICD therapies was as high as $50 \%$ after 5 years, as opposed to $28 \%$ in the study herein.

Other factors are also not especially analyzed in this paper, as e.g. metabolic syndrome with or without obesity, were not shown to influence mortality [13].

To identify predictors of ICD therapy, early or rather late after implantation, is less useful in daily life. This is because a) there are data [7] showing that a substantial proportion of patients have 
their first ICD therapy after 5 years and b) especially in secondary prevention guidelines regarding continuation of ICD treatment even in patients without ICD therapy for many years are clear-cut. Nevertheless, this issue has been investigated herein. In the present CRT-D population, only two predictors could be identified (after analysing 33 parameters) for early ICD therapy, secondary prevention (which is commonly known) and renal failure. A Dutch cohort study [8] did not find a predictor using eight parameters. Identifying predictors that somehow "protect" patients from early or late ICD therapy seems alluring at first glance, as one could argue that such a patient might not be in need of the ICD part of CRT. Further studies especially in patients with dilated cardiomyopathy are needed that include parameters not used in the present dataset such as a true left bundle branch block as compared to other forms of QRS widening or late gadolinium enhancement as seen on magnetic resonance imaging.

\section{Limitations of the study}

This study has all the limitations of a retrospective database study. There was no "control group" with patients with only CRT-P, who might have had other predictors for early death other than CRT-D patients. Some data had to be imputed. Finally, about $10 \%$ were lost to follow-up, and in about the same percentage, the mode of death was unknown. This does, however, not influence prediction of early mortality, as the analysis was performed regarding all-cause mortality.

\section{Conclusions}

Predictors for early mortality after CRT-D implantation were a history of PCI and PVD, however present in only a minority of patients. A survey of the available literature suggests that it is difficult to predict early mortality, albeit this would impact on those patients with a high chance of dying and have no benefit from the ICD part of CRT.

Conflict of interest: Simon von Gunten and Tobias Reichlin: none declared; Dominic A. Theuns: Biotronik: research funding, Boston Scientific: research funding, consultant; Michael Kühne: Medtronic: proctor; Christian Sticherling: Biotronik: speaker bureau, consultant, investigator, research funding; Boston Scientific: investigator; Medtronic: investigator, advisory board; Microport: speaker bureau, consultant; Beat Schaer: Medtronic: speaker bureau; Microport: speaker bureau

\section{References}

1. Cleland JGF, Daubert JC, Erdmann E, et al. The effect of cardiac resynchronization on morbidity and mortality in heart failure. N Engl J Med. 2005; 352(15): 1539-1549, doi: 10.1056/NEJMoa050496, indexed in Pubmed: 15753115.

2. Tang ASL, Wells GA, Talajic M, et al. Cardiac-resynchronization therapy for mild-to-moderate heart failure. N Engl J Med. 2010; 363(25): 2385-2395, doi: 10.1056/NEJMoa1009540, indexed in Pubmed: 21073365.

3. Daubert JC, Martins R, Leclercq C, et al. Why we have to use cardiac resynchronization therapy-pacemaker more. Card Electrophysiol Clin. 2015; 7(4): 709-720, doi: 10.1016/j. ccep.2015.08.016, indexed in Pubmed: 26596813.

4. Kraaier K, Scholten MF, Tijssen JGP, et al. Early mortality in prophylactic implantable cardioverter-defibrillator recipients: development and validation of a clinical risk score. Europace. 2014; 16(1): 40-46, doi: 10.1093/europace/eut223, indexed in Pubmed: 23918791.

5. Theuns DA, Schaer BA, Soliman OII, et al. The prognosis of implantable defibrillator patients treated with cardiac resynchronization therapy: comorbidity burden as predictor of mortality. Europace. 2011; 13(1): 62-69, doi: 10.1093/europace/euq328, indexed in Pubmed: 20833692.

6. Proclemer A, Muser D, Campana A, et al. Indication to cardioverterdefibrillator therapy and outcome in real world primary prevention. Data from the IRIDE [Italian registry of prophylactic implantation of defibrillators] study. Int J Cardiol. 2013; 168(2): 1416-1421, doi: 10.1016/j.ijcard.2012.12.042, indexed in Pubmed: 23287697.

7. Reichlin T, Kühne M, Sticherling C, et al. Characterization and financial impact of implantable cardioverter-defibrillator patients without interventions 5 years after implantation. QJM. 2011; 104(10): 849-857, doi: 10.1093/qjmed/hcr081, indexed in Pubmed: 21624895.

8. Ypenburg C, van Erven L, Bleeker GB, et al. Benefit of combined resynchronization and defibrillator therapy in heart failure patients with and without ventricular arrhythmias. J Am Coll Cardiol. 2006; 48(3): 464-470, doi: 10.1016/j.jacc.2006.04.072, indexed in Pubmed: 16875970.

9. Rubin DB, Schenker N. Multiple imputation in health-care databases: an overview and some applications. Stat Med. 1991; 10(4): 585-598, indexed in Pubmed: 2057657.

10. Kramer DB, Friedman PA, Kallinen LM, et al. Development and validation of a risk score to predict early mortality in recipients of implantable cardioverter-defibrillators. Heart Rhythm. 2012; 9(1): 42-46, doi: 10.1016/j.hrthm.2011.08.031, indexed in Pubmed: 21893137.

11. Ertel D, Phatak K, Makati K, et al. Predictors of early mortality in patients age 80 and older receiving implantable defibrillators. Pacing Clin Electrophysiol. 2010; 33(8): 981-987, doi: 10.1111/j.15408159.2010.02729.x, indexed in Pubmed: 20230459.

12. Husaini M, Biton Y, Stair B, et al. Effectiveness of cardiac resynchronization therapy by the frequency of revascularization procedures in ischemic cardiomyopathy patients. Cardiol J. 2016; 23(4): 437-445, doi: 10.5603/CJ.a2016.0032, indexed in Pubmed: 27320956.

13. Szepietowska B, McNitt S, Polonsky B, et al. Metabolic syndrome is associated with different clinical outcome after cardiac resynchronization therapy in patients with ischemic and non-ischemic cardiomyopathy. Cardiol J. 2016; 23(3): 344-351, doi: 10.5603/CJ.a2016.0017, indexed in Pubmed: 27064797. 